\title{
Adaptive and degenerative evolution of the S-Phase Kinase- Associated Protein 1-Like family in Arabidopsis thaliana
}



Genome sequencing has uncovered tremendous sequence variation within and between species. In plants, in addition to large variations in genome size, a great deal of sequence polymorphism is also evident in several large multi-gene families, including those involved in the ubiquitin-26S proteasome protein degradation system. However, the biological function of this sequence variation is yet not clear. In this work, we explicitly demonstrated a single origin of retroposed Arabidopsis Skp1-Like (ASK) genes using an improved phylogenetic analysis. Taking advantage of the 1,001 genomes project, we hereinto provide several lines of polymorphism evidence showing both adaptive and degenerative evolutionary processes in ASK genes. Yeast two-hybrid quantitative interaction assays further confirmed that recent neutral changes in the ASK2 coding sequence weakened its interactions with some F-box proteins. The trend that highly polymorphic upstream regions of ASK1 yield high levels of expression implied negative expression regulation of ASKI by an as-yet-unknown transcriptional suppression mechanism, which may contribute to the polymorphic roles of Skp1-CUL1-F-box complexes. Taken together, this study provides new evolutionary evidence to guide future functional genomic studies of SCF-mediated protein ubiquitylation. 
8

23

24 25 26

27

\section{Adaptive and Degenerative Evolution of the S-Phase Kinase-Associated Protein 1-Like} Family in Arabidopsis thaliana

Zhihua Hua ${ }^{1}$ and Zhenyu Gao ${ }^{1,2}$

${ }^{1}$ Department of Environmental and Plant Biology and Interdisciplinary Program in

Molecular and Cellular Biology, Ohio University, Athens, Ohio 45701, USA;

2State Key Laboratory of Rice Biology, China National Rice Research Institute, Hangzhou 310006, Zhejiang, China

Running title: Evolution of the Arabidopsis Skp1-like family

*Corresponding author: Zhihua Hua, Department of Environmental and Plant Biology, Ohio University, Athens, Ohio 45701 USA. Tel: +01740 593 1123, Email: hua@ohio.edu 


\section{Abstract}

29 Genome sequencing has uncovered tremendous sequence variation within and between

30 species. In plants, in addition to large variations in genome size, a great deal of sequence

31 polymorphism is also evident in several large multi-gene families, including those involved in the

32 ubiquitin-26S proteasome protein degradation system. However, the biological function of this

33 sequence variation is yet not clear. In this work, we explicitly demonstrated a single origin of

34 retroposed Arabidopsis Skp1-Like (ASK) genes using an improved phylogenetic analysis.

35 Taking advantage of the 1,001 genomes project, we hereinto provide several lines of

36 polymorphism evidence showing both adaptive and degenerative evolutionary processes in

37 ASK genes. Yeast two-hybrid quantitative interaction assays further confirmed that recent

38 neutral changes in the ASK2 coding sequence weakened its interactions with some F-box

39 proteins. The trend that highly polymorphic upstream regions of $A S K 1$ yield high levels of

40 expression implied negative expression regulation of $A S K 1$ by an as-yet-unknown

41 transcriptional suppression mechanism, which may contribute to the polymorphic roles of Skp1-

42 CUL1-F-box complexes. Taken together, this study provides new evolutionary evidence to

43 guide future functional genomic studies of SCF-mediated protein ubiquitylation. 


\section{Introduction}

45 Proteins play fundamental roles in driving life processes by sensing diverse environmental cues, 46 catalyzing biochemical reactions, monitoring the stability of genetic materials, and combating 47 abiotic and biotic stresses. In addition, they are believed to be the only molecules capable of 48 mechanical movement in any organism. To accomplish these diverse roles, not only is protein 49 synthesis precisely controlled, but the structure, activity, and turnover of each protein is also 50 sophisticatedly regulated in a temporal and spatial manner. The ubiquitin-26S proteasome 51 system (UPS) is the primary degradative machinery for rapidly modulating protein content in 52 eukaryotic cells. Given the power of its selective turnover of numerous intracellular proteins, the 53 UPS plays an essential regulatory role in controlling cell cycle progression, signal transduction, 54 gene expression regulation, genome stability, and many other cellular processes (Finley et al. 55 2012; Vierstra 2009; Yau and Rape 2016). This function has been demonstrated to be 56 particularly important in plants, as evidenced by the extremely large expansion of several gene superfamilies that encode plant UPS members (Vierstra 2009).

58

Among these members, S-Phase Kinase-Associated Protein 1 (Skp1), F-box proteins, Cullin 1 and RING box protein 1 (RBX1) compose the largest group of ubiquitin-ligase complexes in plants, termed SCF complexes, which target protein substrates for ubiquitylation and subsequent turnover by the 26S proteasome (Hua and Vierstra 2011). Genetic, genomic, evolutionary, and biochemical analyses have shown that the F-box multi-gene superfamily encodes a substrate receptor that determines the specificity of the SCF complex, while the Skp1 protein family functions as an adaptor to bridge the variable F-box proteins to the N-terminus of Cullin 1 to assemble a holo-ubiquitin ligating enzyme (Gagne et al. 2002; Hua and Vierstra 2011; Yang et al. 1999; Zheng et al. 2002). Although a handful of F-box proteins have been functionally shown to target the degradation of proteins involved in the cell cycle, circadian rhythms, photomorphogenesis, pathogen defense, hormone signaling, and plant reproduction, many recently duplicated and species- or lineage-specific members remain uncharacterized (Hua et al. 2011). Given that the size of the F-box superfamily is species-specific and is often not correlated with the complexity of plant species, a genomic drift evolutionary mechanism has been postulated to explain the random size drift of the F-box gene superfamily in plants (Hua et al. 2011; Nozawa et al. 2007; Xu et al. 2009). The high sequence polymorphism of lineage specific F-box genes and their enrichment of transcriptional suppression-related epigenetic modifications further support this hypothesis (Hua et al. 2013). However, this does not preclude 
78 (Gagne et al. 2002; Shabek and Zheng 2014; Yang et al. 2008). Unfortunately, it remains 79 difficult to find these members both experimentally and theoretically, in part due to the large size 80 of this group and the low/no expression of most lineage specific F-box genes (Hua et al. 2013). 81

82 Similar to the F-box gene superfamily, the Skp1 family has also expanded significantly in land 83 plants. While there is only one single Skp1 protein encoded in yeast and human genomes, the 84 genomes of Arabidopsis thaliana and Oryza sativa contain 21 and 32 annotated Skp1 loci, 85 respectively (Kong et al. 2007). Cross-kingdom evolutionary studies have suggested that the 86 plant Skp1 genes are also rapidly evolving through a birth-and-death evolutionary mechanism 87 (Kong et al. 2004; 2007). However, unlike many inactive F-box genes (Hua et al. 2011; Kuroda 88 et al. 2012), 20 out of 21 A. thaliana Skp1-Like (ASK) genes are transcribed in at least one out 89 of 6 tissues/organs examined, including seedlings, roots, stems, leaves, inflorescences, and 90 siliques (Kong et al. 2004; Zhao et al. 2003), suggesting that most ASK genes are active.

91 Phylogenetic analysis further implied that all plant Skp1 genes shared one common ancestor, 92 although evolutionary rates of individuals are highly heterogeneous. Therefore, it has been 93 inferred that some moderately and rapidly evolving members might have lost their original 94 functions and/or gained new functions (Kong et al. 2004). Despite the sequence diversity of 95 ASK proteins, a recent biochemical study showed that all ASK proteins retained the biochemical function of their ancestor Skp1 protein for interacting with F-box proteins (Kuroda et al. 2012).

The fact that all ASK proteins interact with an F-box protein implied that their sequences are not 99 sufficiently diverged from their ancestor sequence for their original biochemical function to have been lost. However, this rapid evolution has dramatically diversified the sequences of plant Skp1 genes, making it challenging to uncover the true phylogenetic relationships among distantly related plant species. Indeed, to avoid the effects of long-branch attraction, type II Skp1 genes, which carry multiple introns in various positions, unlike type I Skp1 genes that contain only one or none introns, were excluded in a previous phylogenetic analysis of Skp1 genes (Kong et al. 2007). Improved understanding of the evolutionary mechanisms of the Skp1 gene family may aid further exploration of the functions of many unknown SCF complexes. To date, the genome sequences of three Arabidopsis species, A. thaliana, A. lyrata, and A. halleri, which split 5-10 million years ago (mya) (Hu et al. 2011; Koch and Kiefer 2005), have been obtained [The Arabidopsis Information Resource (TAIR), V10; Phytozome V12], and >1,000 individual $A$. thaliana accessions have been sequenced (Consortium 2016). These datasets can allow us to further fine-tune the phylogenetic relationships and fixation processes of rapidly- 
112 evolving genes in plants, which may help better define their functional constraints. Because the

113 diverse functions of the SCF complexes are primarily determined by the large F-box gene family

114 along with the Skp1 gene family, in this work we analyzed the short evolutionary history of the

115 Skp1 genes within and between Arabidopsis species in order to uncover important evolutionary

116 patterns in SCF regulatory pathways. Our new evidence suggests that the ASK genes are

117 under both adaptive and degenerative evolutionary processes.

118

119 Materials and Methods

120 Identification of Skp1 genes in A. lyrata and A. halleri

121 The full set of Skp1 seed sequences that encompass a 70 to 86 amino acid core Skp1 domain

122 were retrieved from Pfam (PF01466, Version 27, http://pfam.xfam.org) and used as query in a

123 BLASTp search (Altschul et al. 1990) against the annotated proteome of each species, which

124 was retrieved from Phytozome (http://phytozome.jgi.doe.gov/; A. lyrata V2 and A. halleri V1.1).

125 The presence of Skp1 and any additional protein-protein interacting domains in each full-length

126 hit sequence were further confirmed by hmmscan (http://hmmer.org) against the Pfam-A

127 database (Pfam 27, http://pfam.xfam.org). To identify a complete list of Skp1 genes in each

128 species, a previously developed sequence similarity-based annotation algorithm, called Closing

129 Target Trimming (Hua and Early 2018; Hua et al. 2011), was also used to search the genomes

130 for any new Skp1 loci that may not have been annotated.

131

132 Sequence alignment and phylogenetic analysis

133 Instead of manual adjustment and artificial deletion of ambiguous alignment as reported in the

134 previous studies (Kong et al. 2004; 2007; Zhao et al. 2003), two Skp1 protein sequence

135 alignments were obtained by MUSCLE (Edgar 2004) and MAFFT (Katoh et al. 2017), and then

136 used to make a consensus alignment by Trimal (-conthreshold 0.5) (Capella-Gutierrez et al.

137 2009). The resulting alignment was used to generate a maximum likelihood phylogenetic tree

138 by RAxML (Version 8.1; Stamatakis 2014) with the PROTGAMMAJTT substitution model. The

139 statistical significance was evaluated with 1,000 bootstrap replicates using a rapid bootstrap

140 analysis.

141

142 Birth and death of the Skp1 genes in the Arabidopsis genus

143 Gene duplication and loss events were inferred by reconciling the maximum likelihood gene tree

144 with the species tree using Notung (version 2.9) (Chen et al. 2000). 


\section{Gene structure analysis and reference sequence retrieval}

147 The number of introns in each Skp1 gene was counted based on the Generic Feature Format

148 (GFF3) file from each genome project. According to the chromosomal coordinates, the upstream 149 and downstream regions of a Skp1 gene, which are 500 nucleotides upstream of the start codon 150 and downstream of the stop codon, respectively, were retrieved from the genomes of $A$. thaliana 151 (TAIR V10; www.arabidopsis.org) and A. lyrata (V2; Rawat et al. 2015). The coding sequence of 152 an ASK gene within the Col-0 reference genome and its $A$. lyrata ortholog was retrieved from the 153 annotated transcriptomes of $A$. thaliana (TAIR V10) and A. lyrata (V2; Rawat et al. 2015), 154 respectively.

155

156 Sequence assembly for polymorphism analysis

157 To assemble an ASK allelic sequence, single-nucleotide polymorphic (SNP) alleles (Phred quality 158 score $\geq 25$ ) within the coding and non-coding flanking sequences were first retrieved from each 159 Arabidopsis accession (http://1001genomes.org). In total, 774 accessions were selected (Cao et 160 al. 2011; Consortium 2016; Long et al. 2013; Schmitz et al. 2013). Based on their co-ordinates, 161 both variant and the Col-0 reference SNP alleles were used to substitute the nucleotides in a 162 reference sequence to assemble two allelic sequences. Only when the new Col-0 allelic 163 sequence was $100 \%$ identical to the reference sequence was the variant allele considered to be 164 assembled correctly. To assemble an outgroup sequence, an amino acid sequence alignment of 165 an ASK protein and its A. lyrata ortholog was obtained by MAFFT (Katoh et al. 2017) and used to 166 guide the assembly of a nucleotide sequence of the outgroup. The sites introducing gaps in the 167 reference $A S K$ sequence were removed.

168

169

\section{Determination of tandemly duplicated Skp1 genes}

170 Two Skp1 genes were determined to be tandemly duplicated if they were both separated by $\leq 5$ 171 genes and located within $10 \mathrm{~kb}$.

172

173 Clustering analysis

174 Sequences were clustered using Heatmap.2 (dist method = "manhattan", hclust method = 175 "word.D") in R (http://www.r-project.org) to demonstrate similar evolutionary constrains of 176 mutations as described previously (Hua et al. 2018).

177

\section{Expression data resources}


179 The RNA-Seq data for leaf or seedling transcriptomes from 144 and 19 different $A$. thaliana 180 accessions were retrieved from the projects published by Schmitz et al. (2013) and Gan et al. 181 (2011), respectively. Microarray expression data of 79 samples collected from 8 different 182 tissues/organs throughout the $A$. thaliana Col-0 life cycle were downloaded from 183 http://jsp.weigelworld.org/expviz/expviz.jsp.

184

\section{Cross species test of neutral evolution}

186 The orthologous pairing of a Skp1 gene between $A$. thaliana and A. lyrata was determined by 187 OrthoMCL (Li et al. 2003) and used to examine its neutral evolutionary process as previously 188 described (Nekrutenko et al. 2002), with minor modifications. The nucleotide sequences of each 189 pair were aligned based on the protein sequence alignment obtained by T-Coffee (Taly et al. 190 2011) and used as an input file to run the codeml program from the PAML4 package (Yang 2007) 191 twice, with the Ka/Ks ratio either fixed at 1 or free. The maximum likelihood (ML) values ML1 and 192 ML2 from the two runs were collected to calculate the likelihood ratio as $L R=2(\operatorname{InML} 1-\operatorname{InML} 2)$. If 193 LR is less than 2.71 (5\% significance for $X 2$ distribution with one degree of freedom) (Yang 2007), 194 the KalKs ratio is considered not significantly different from 1, i.e. the Skp1 gene is under a neutral 195 evolutionary process.

196

197 Molecular cloning and yeast two-hybrid analysis

198 The coding sequences of 15 selected known F-box genes and ASK1/2 were PCR amplified 199 from cDNA clones that were obtained from the Arabidopsis Biological Resource Center 200 (https://abrc.osu.edu) and ligated in-frame to the 3'-end of GAL4-BD and GAL4-AD coding 201 sequences present in the yeast two-hybrid vectors, pGBK-T7 (bait) and pGAD-T7 (prey), 202 respectively. The resulting bait and prey vectors were separately transformed into the haploid 203 yeast strains, $\mathrm{AH} 109$ and $\mathrm{Y} 187$, respectively, which were subsequently mated according to the 204 pairwise interaction combinations.

205

206 For yeast growth interaction assays, the number of mated diploid yeast cells were normalized 207 and diluted with sterile water in series to an $\mathrm{OD}_{600}$ of $0.8,0.4,0.2$ and $0.1 .5 \mu \mathrm{L}$ of yeast cells 208 from each concentration were then spotted on either a quadruple synthetic dropout medium 209 (SD-Leu-Trp-Ade-His) containing X-a-gal $(40 \mu \mathrm{g} / \mathrm{mL})$ for interaction assays, or on a double 210 synthetic dropout medium (SD-Leu-Trp) as a growth control. To quantify the interaction

211 strength, the intensity of yeast growth from the scanned images was calculated using 
212 ImageQuant version 5.2 (GE Healthcare). Each interaction signal on SD-Leu-Trp-Ade-His+X- $\alpha-$

213 gal medium was normalized to that detected on SD-Leu-Trp medium.

214

215 For the $\beta$-galactosidase activity assay, six to ten mated yeast colonies grown on SD-Leu-Trp

216 medium were freshly harvested and resuspended in $0.5 \mathrm{~mL}$ of $Z$ buffer $(50 \mathrm{mM}$ sodium

217 phosphate, $10 \mathrm{mM}$ potassium chloride, $2 \mathrm{mM}$ magnesium sulfate, $\mathrm{pH} 7.0$ ) in a $2 \mathrm{~mL}$ deep-well

218 plate. $0.1 \mathrm{~mL}$ of resuspended yeast cells were further aliquoted into a new $2 \mathrm{~mL}$ deep-well plate

219 and used for $\beta$-galactosidase activity according to Miller (Miller 1972). Relative $\beta$-galactosidase

220 activities were calculated based on the method described previously (Hua and Kao 2006). In

221 total, two independent replicates were assayed.

222

\section{Results}

\section{Identification of Skp1 genes in three closely related Arabidopsis species}

225 The list of 21 A. thaliana ASK genes, which contain 19 Type I (ASK1-19) and 2 Type II (ASK20

226 and 21) Skp1 genes, has been well annotated in previous studies (Farras et al. 2001; Kong et

227 al. 2007; Kong et al. 2004; Zhao et al. 2003). However, a full list of Skp1 genes has not been

228 reported in A. lyrata or A. halleri. To identify a comprehensive list of Skp1 genes in these two

229 Arabidopsis genomes, BLASTp (Altschul et al. 1990) and hmmscan (http://hmmer.org) were first

230 applied to search the available genome annotations [A. lyrata V2 (Rawat et al. 2015), A. halleri

231 V1.1 (Phytozome V12)]. In total, 17 and 11 loci were identified that encode a Type I Skp1

232 protein, and 4 and 3 loci were discovered to encode a Type II Skp1 protein, in $A$. lyrata and $A$.

233 halleri, respectively (Supplemental File S1). After a subsequent sequence-similarity based

234 Closing Target Trimming search (Hua and Early 2018; Hua et al. 2011), no additional hits were

235 identified in each genome. The relatively long length of the Skp1 domain, the low number or

236 absence of introns in the Skp1 loci, and the small size of the Skp1 family, may facilitate the

237 annotation of Skp1 genes in genomes. Therefore, in total 21, 21, and 14 Skp1 loci are present

238 in A. thaliana, A. lyrata, and A. halleri, respectively. The size variation among these closely

239 related Arabidopsis species indicates that the Skp1 family is, like the F-box gene superfamily,

240 under a rapid birth-and-death evolutionary process.

241

242 Phylogenetic analysis of the Skp1 genes in a short evolutionary history

243 Since the sequences of Type II Skp1 genes are significantly diverged from Type I Skp1 genes,

244 and most Skp1 genes are Type I (Kong et al. 2007), hereafter we focused on the evolutionary

245 study of the Type I group. To understand the birth-and-death process of the Skp1 family in a 
246 short evolutionary history, we performed a phylogenetic analysis using an improved sequence

247 alignment approach. Since manual adjustment and artificial deletion of ambiguous alignment

248 sites are not always reproducible, Trimal (Capella-Gutierrez et al. 2009) was used to remove

249 poorly aligned regions automatically. In addition, a consensus result was obtained from

250 MUSCLE (Edgar 2004) and MAFFT (Katoh et al. 2017) sequence alignments to improve

251 accuracy (see Materials and Methods). The resulting alignment not only significantly reduced

252 gaps and mis-matched sites, but also retained a reproducible result with $95 \pm 5 \%$ of the full

253 length Skp1 protein sequences being aligned (Figure S1), maximizing the sequence length and

254 variable sites essential for a good phylogenetic analysis (Nei and Kumar 2000). As a proof of

255 concept, an ASK2-rooted maximum likelihood tree generated based on the 19 aligned ASK

256 protein sequences showed a compatible topology to the one reported previously (Kong et al.

257 2007) (Figure 1A). However, unlike the previous tree where intronless and intronic ASK genes

258 are intermingled (Kong et al. 2007), all intron-containing ASK genes (except for ASK15, whose

259 intron was gained after duplication (Kong et al. 2007)) were clustered at the base of the tree

260 (Figure 1A). This result better explains a single origin of the intronless ASK genes, which were

261 duplicated through retroposition from a highly expressed ASK gene, most likely the ancestor of

262 ASK1.

263

264 Subsequently, a maximum likelihood tree rooted to ASK2 was generated based on the

265 consensus protein sequence alignment of the 47 Arabidopsis Skp1 protein sequences by

266 RAxML (Stamatakis 2014). Encouragingly, the resulting phylogenetic tree showed a similar

267 topology to that obtained for the ASK genes, and the 47 Skp1 genes from the three Arabidopsis

268 species were intermingled in 11 clusters (Figure 1B). Therefore, most Arabidopsis Skp1 genes

269 were duplicated at least 5-10 mya, at the time when the three Arabidopsis species split (Hu et

270 al. 2011; Koch and Kiefer 2005). Similarly to the ASK genes, all intronic Arabidopsis Skp1

271 genes were clustered at the base of the tree, while the remaining intronless Skp1 genes were

272 clustered into one big clade, suggesting a common role of retroposition in the expansion of the

273 Skp1 family in Arabidopsis. Among these, three ancestor loci (Figure 1B, nodes a, $c$, and $d$ )

274 were likely duplicated through retroposition, with each likely further undergoing tandem

275 duplication events to yield the current Skp1 members. Two Skp1 clades (Figure 1B, clades 2

276 and 6) were likely the direct product of a retroposition event. Therefore, the mRNAs produced

277 by the highly expressed $A S K 1$ ancestor were likely retrotransposed to 5 ancestor loci in total

278 (Figure 1B, nodes a-e). This phylogenetic tree also showed a clear duplication event between

279 the ASK1 clade and the ASK4 clade before the split of three Arabidopsis species. Although 
280 Kong et al. (2007) first reported the contribution of segmental duplication in duplicating the

281 ASK1 and ASK4 loci, their phylogenetic tree did not reflect the direct connection between these

282 two genes (Kong et al. 2007).

283

284

285

We further reconciled a gene tree based on this maximum likelihood tree. Along with a species

286 tree, we detected significant variance of birth/death rates between each species (Figure 1C). While A. halleri lost 7 Skp1 loci after a recent split from A. lyrata, $A$. thaliana has gained and lost 5 loci each from the 19 common Arabidopsis Skp1 gene ancestors. The birth/death rate of Skp1 genes in A. lyrata was intermediate among the three species; it gained and lost 2 and 4 Skp1 genes, respectively, following the split from $A$. thaliana. Such a significant size variation even within a short evolutionary history implied that some ancestral retroposed Skp1 loci resided in a hot spot of tandem duplications, which contributed to the differential sizes of the Skp1 family among the three Arabidopsis species. For example, at the ancestor "a" locus, 4 and 3 Skp1 genes were gained through tandem duplications within the past 5-10 million years in

295

\section{Low evolutionary constraints of intronless ASK genes}

297 The phylogenetic tree revealed a clear orthology relationship between the Skp1 genes of the 298 three Arabidopsis species. To further demonstrate this relationship, we applied OrthoMCL (Li et al. 2003) to identify 14 Skp1 orthologous groups, among which 18 ASK genes have been partnered with one A. lyrata Skp1 orthologous gene (Supplemental Table S1). This clear orhology relationship allowed us to examine evolutionary constraints on the sequence divergence of ASK genes. We primarily applied the method of Nekrutenko et al. (2002) to test whether the $K a / K s$ ratio (i.e., $\omega$ ) of an $A S K$ gene is significantly diverged from 1 , which indicates between-species neutral nucleotide divergence. Surprisingly, 9 out of 14 (64\%) intronless ASK genes were detected to be under a neutral evolutionary process, while none of the 4 intronic ASK genes belongs to this category (Table 1), suggesting that the former group has lower functional constraints than the latter. Interestingly, using GeneWise reannotation (Birney et al. 2004), we identified a frame-shift mutation in the ASK6, AlySkp02, and AhaSkp11 loci that is characteristic of pseudogenes (Figures S2-S4).

\section{Expression variation of the ASK genes}

312 Low functional constraints do not necessarily mean no function (Nei and Kumar 2000). To 313 understand the functional differences between ASK genes, previous studies examined their 
314 expression patterns in different tissues/organs by semi-quantitative reverse transcription-PCR

315 (RT-PCR) and in situ hybridization (Kong et al. 2004; Zhao et al. 2003). The results suggested

316 that all ASK genes could be expressed in at least one of six samples examined (mostly in

317 siliques). However, only the highly-expressed ASK1 and ASK2 genes showed a consistent

318 result in both studies, while other ASK genes with low expression levels were not stably

319 detected by RT-PCR, probably due to technical limitations (Kong et al. 2004; Zhao et al. 2003).

320 Since then, a number of high throughput transcriptomic analyses, including microarray and

321 RNA-Seq experiments, have been performed in $A$. thaliana, providing good resources to further

322 examine the expression patterns of ASK genes in a more statistical manner.

323

324 Microarray experiments from 79 A. thaliana samples collected from different developmental

325 stages confirmed that $A S K 1$ and $A S K 2$ are the two most highly expressed ASK genes, followed

326 by ASK3. Unlike the previous studies (Kong et al. 2004; Zhao et al. 2003), not all ASK genes

327 were detected in these microarray transcriptomic analyses, probably due to the low sensitivity

328 and precision of microarray technologies (Wang et al. 2009) (Figure 2A). After comparing the

329 expression levels of $A S K$ genes in 8 different tissues/organs (79 samples in total), we found that

330 several $A S K$ genes, including $A S K 1,2,3,7,8,9$, and 10, were expressed most highly in seeds

331 (Figure 2B), suggesting an important role for SCF-mediated protein ubiquitylation in seed

332 development.

333

334 We further analyzed the expression variance of $A S K$ genes at the population level. Based on

335

336

337

338

339

340

341

342

343

344

345

346

347

one RNA-Seq experiment (Schmitz et al. 2013), which provided a much more precise and sensitive transcriptomic analysis than microarrays (Wang et al. 2009), the transcripts of each ASK gene could be detected in leaf tissues of $144 \mathrm{~A}$. thaliana accessions, but with dramatic variance (Figure $2 \mathrm{C}$ ). Consistent with the previous studies and the microarray data, ASK1 has the highest expression level, followed by ASK2. However, the remaining ASK genes were only expressed at an average of 1.3 Reads Per Kilobase of transcript per Million mapped reads (RPKM), 121- and 42-fold below the mean expression of ASK1 and ASK2, respectively. Therefore, while $A S K 1$ and $A S K 2$ likely have important function(s), the role(s) of the other $A S K$ genes in leaves appears minor. Interestingly, the four $A S K$ genes ( $A S K 1,2,4$, and 18) whose mean expression levels are significantly higher than the other ASK genes (Wilcoxon rank- sum test, $p<2.2 \mathrm{e}-16$,) are all under strong evolutionary constraints, and three of them are intronic, further supporting our conclusion regarding the low functional constraints of intronless ASK genes.

Peer] reviewing PDF | (2019:02:34819:1:1:NEW 2 Mar 2019) 
349 In addition to the expression variation among different ASK genes, the expression of ASK1 and 350 ASK2 varied dramatically among different individuals. For example, the highest and the lowest 351 expression levels of ASK1 were detected in the Gr-1 (480 RPKM, Longitude/Latitude/Altitude = 352 15.5/47/300) and Co-1 (50 RPKM, Longitude/Latitude/Altitude = -8.3/40.1/100) accessions, 353 respectively, varying by 430 RPKM, while the highest and the lowest expression levels for ASK2 354 were detected in Ven-1 (210 RPKM, Longitude/Latitude/Altitude = 5.6/52/parking lot) and Ann-1 355 (10 RPKM, Longitude/Latitude/Altitude = 6.1/45.9/garden), respectively, differing by 200 RPKM 356 (Figure 2C). To confirm this result, we also examined the differential expression of ASK1 and 357 ASK2 in another RNA-Seq experiment (Gan et al. 2011). The expression of ASK1 varied 358 significantly among Col-0, Ler-0, and Ws-0, which are from distinct geographic regions, while 359 ASK2 showed only mild changes (Figure 2D). Therefore, although ASK1 has the highest

360

361

362

363

364

365

366

367

368

369

370

371

372

373

374

375

376

377

378

379 380 expression level among all the ASK genes, its expression varies the most among individuals, reflecting a possible adaptation of its expression regulation.

\section{Differential sequence polymorphism of the ASK genes}

We further examined sequence diversity and polymorphism of the $A S K$ genes within the family by comparing number of segregating sites per nucleotide site $(\theta)$ and nucleotide diversity $(\pi)$ values in the regions $500 \mathrm{bp}$ upstream of the start codon, within the coding sequence, and 500 bp downstream of the stop codon of an ASK gene among $774 \mathrm{~A}$. thaliana accessions based on their single nucleotide polymorphic (SNP) data (Consortium 2016). We first calculated the allele frequency distribution and noticed that minor allele frequency (MAF) alleles were significantly enriched. In the total populations analyzed, $34 \%$ of non-synonymous alleles and $35 \%$ synonymous alleles are only present once (i.e., singleton) in the ASK family (Figure 3A), suggesting that many mutations are rare.

74 Consistent with the enrichment of low MAF alleles, the $\pi$ values are significantly lower than the $\theta$ values in coding sequences (Figure 3B), because the former is determined by allele frequency and the latter is not (Nei and Kumar 2000). Similar to the coding sequences, the $\pi$ values are also significantly lower than the $\theta$ values in both upstream and downstream regions, indicating that low MAF alleles are also high in these two regions. As expected, these two regions have higher $\pi$ and $\theta$ values than the coding sequences due to their low functional constraints. 
381 The variance of $\pi$ and $\theta$ values within and between different regions of the ASK genes indicates

382 different extents of polymorphism. To further examine the evolutionary constraints of

383 polymorphic mutations, a Tajima's D value (Tajima 1989) was calculated for the three regions of

384 an ASK gene (upstream, coding sequence (CDS) and downstream). According to the variance

385 of this value, the 18 ASK genes as described in Table 1 were clustered into 3 groups (Figure

386 4C). In Group I, which contains ASK4, 6, 10, 14, and 19, a high Tajima's D value was observed

387 in the coding sequences, suggesting a balancing or neutral evolutionary process. Group II,

388 which includes $A S K 5,8,9,12,13,16$, and 17, shows intermediate Tajima's D values, some of

389 which are significantly smaller than the Tajima's critical values of neutral mutations $(p<0.05)$

390 (Tajima 1989), suggestive of purifying selection. The remaining 6 ASK genes (ASK1, 2, 3, 7,

391 11, and 18) are clustered into Group III where most have a Tajima's D value below the Tajima's

392 critical values of neutral mutations in all three regions $(p<0.05)$. Therefore, mutations in this

393 group are rare, and most sequences are under strong purifying selection. Interestingly, this

394 group enriched 5 out of 6 ASK genes that were detected to be under non-neutral changes by

395 orthology comparison (Table 1), further confirming their high evolutionary constrains. It is worth

396 noting that the Tajima's D value of the coding sequence of ASK2 is within the range of Tajima's

397 critical values, suggesting that most polymorphic SNPs in ASK2 are neutral.

398

399 Variation association of ASK1 expression and polymorphism

400 To associate sequence polymorphism with gene expression variation, we compared the

401 expression patterns and pair-wise nucleotide diversity ( $\pi)$ of $144 \mathrm{~A}$. thaliana accessions from

402 the RNA-Seq experiment done by Schmitz et al. (2013). Based on the wide latitudinal

403 distribution of accessions, 5 subgroups were separated (Figure 4A). Although no linear

404 regression is observed between individual expression levels and latitudes, accessions within

405 latitudes $45-50^{\circ} \mathrm{N}$ have significantly higher levels of $A S K 1$ gene expression than the two flanking

406 regions $5^{\circ}$ to the north or south (Wilcoxon rank-sum test, $p<0.01$ ). Since 116 out of 144

407 accessions (81\%) reside in these three sub-regions, the populations in these areas were further

408 analyzed. To better understand the relationship between nucleotide diversity and expression

409 levels of $A S K 1$, the entire $2.5 \mathrm{~kb}$ intergenic sequence upstream of the transcription start site

410 (TSS) of ASK1 was compared. A window-sliding analysis (200 bp window and $100 \mathrm{bp}$ slide)

411 showed that the windowed $\pi$ values from nucleotide -1600 to -500 are significantly higher in

412 accessions within latitudes $45-50^{\circ} \mathrm{N}$ than those in the other two regions (Figure 4B, Wilcoxon

413 rank-sum test, $p<0.05)$. Such a relationship between high polymorphism and high expression

414 suggests that nucleotide variance in the promoter may prevent the binding of a putative

Peer] reviewing PDF | (2019:02:34819:1:1:NEW 2 Mar 2019) 
415 transcriptional repressor that may suppress the expression of ASK1. To further demonstrate

416 that the accessions within latitudes $45-50^{\circ} \mathrm{N}$ are highly polymorphic in the promoter region, we

417 also compared accessions from the projects in Cao et al. (2011) and Long et al. (2013). In total,

41829,20 , and 12 accessions resided in latitudes $40-45,45-50$, and 50-60 $\mathrm{N}$, respectively.

419 Consistently, the accessions from latitudes $45-50^{\circ} \mathrm{N}$ also have the highest sequence

420 polymorphism from nucleotide -1600 to -500 among the three groups compared (Figure 4C).

421 We also applied a different grouping method by separating the aforementioned 144 accessions

422 into low (49 accessions with ASK1 expression value in the 50-137 RPKM range), medium (50

423 accessions with ASK1 expression of 137-170 RPKM), and high (45 accessions with ASK1

424 expression of 170-480 RPKM) expression groups. Among these three groups, high nucleotide

425 polymorphism in the -1000 to -500 region is also most evident in the group with high ASK1

426 expression (Figure 4D).

427

\section{Variance of biochemical interactions of ASK1 and ASK2 with known F-box proteins}

429 The different extent of sequence variation between ASK1 and ASK2 coding sequences (Figure

$4303 \mathrm{C}$ ) led us to speculate that their encoded proteins might show differential strength of interaction

431 with F-box proteins. To address this question, we tested the interactions of ASK1 and ASK2 by

432 pair-wise yeast two-hybrid assay with 15 randomly-selected F-box proteins whose functions

433 have been identified (Figure 5 and Table S2). Due to the identification of neutral polymorphic

434 mutations in the ASK2 CDS and purifying selection in the ASK1 CDS, we hypothesized that

435 ASK2 might have lost or reduced its interactions with a number of F-box proteins. To provide a

436 starting point to examine the potential biochemical differences between ASK1 and ASK2, we

437 performed both yeast growth assay on quadruple synthetic dropout medium (SD-Leu-Trp-Ade-

438 His) containing $X$ - $\alpha$-gal (Figure $5 \mathrm{~A}$ and $5 B$ ) and $\beta$-galactosidase activity analysis (Figure $5 C$ ).

439 Interestingly, we detected that two F-box proteins (JMJ22 and DIF) interacted with ASK1 with a

440 strength $>2$ fold more than with ASK2 (Figure 5B and 5C). However, the remaining F-box

441 proteins showed an average of $15 \%$ variation in their interactions with ASK1 and ASK2,

442 suggesting that the ASK2 protein, albeit carrying recently neutral mutations, still retains the

443 capability in binding many functional F-box proteins. It is worth mentioning that the interaction

444 strengths of ASK1 or ASK2 with different F-box proteins also vary dramatically. This is

445 consistent with the high sequence divergence of F-box genes (Hua et al. 2013).

446

447 Discussion 
448 Gene duplication has been a long-standing topic of interest in genome evolution (Ohno 1970).

449 In eukaryotic genomes, this process plays an essential role in the expansion of many multi-gene

450 families including those involved in the ubiquitin-26S proteasome system (Hua et al. 2011;

451 2013; 2018; Hurles 2004; Li et al. 2016; Panchy et al. 2016). While gene duplication provides

452 the raw genetic material for genome innovation, the large size of multi-gene families has been a

453 hurdle in exploring genome function. For example, it is not yet clear whether and how multiple

454 ASK members contributed to evolutionary innovation through sub-functionalization or neo-

455 functionalization, or whether the expansion of the ASK family is simply due to the selective

456 advantage of gene dosage or is a result of genomic drift. In this study, we applied several novel

457 approaches to address these questions, as outlined below.

458

459 Retroposed ASK genes originated from one single ancestor locus

460 Instead of cross kingdom long-distant phylogenetic studies (Kong et al. 2004; 2007), we focused

461 on a short evolutionary history within the Arabidopsis genus, so that the orthology relationships

462 and duplication history of individual ASK members could be more clearly illustrated. In addition,

463 more advanced sequence alignment tools have been adopted to improve the phylogenetic

464 analysis (Figure 1). For example, although Kong et al. (2007) discovered that ASK1 and ASK4

465 were duplicated through a segmental duplication event by comparing whole genome duplication

466 blocks, their phylogenetic analysis did not reflect this duplication event. Here, we provided

467 improved phylogenetic evidence not only showing the segmental duplication relationship

468 between $A S K 1$ and $A S K 4$, but also demonstrating a single origin of all intronless $A S K$ members

469 from the ancestor of $A S K 1$ through retroposition (Figure 1). This new discovery is also

470 consistent with the high expression of $A S K 1$ (Figure 2) and the young evolutionary history of

471 intronless ASK members. Similarly, a previous study on the placental mammalian Gli-Kruppel

472 type zinc finger transcription factor $Y Y 1$ family revealed that two retroposed intronless

473 subfamilies, YY2 and Reduced Expression 1 (REX1), were separately clustered but not mingled

474 with the intronic YY1 members (Kim et al. 2007).

475

476 Degenerative processes of $A S K$ genes

477 Since the first draft genome of $A$. thaliana was released, the evolutionary process of both the $F$ -

478 box and ASK families has been a hot topic in plant biology due to the importance of SCF-

479 mediated protein ubiquitylation and the unequal expansion of the two families. Although it has

480 been hypothesized that variant ASK proteins might contribute to interactions with a specific

481 group of F-box proteins (Gagne et al. 2002; Kuroda et al. 2012), in vivo data is currently lacking. 
482 Indeed, most functionally characterized F-box proteins physically interact with ASK1 (Hua and 483 Vierstra 2011). Furthermore, recently evolutionary studies have suggested that the number of 484 active F-box members is much fewer than the size of the family, due to a genomic drift 485 evolutionary process (Hua et al. 2011; Nozawa et al. 2007; Xu et al. 2009). This raised a 486 question as to whether all ASK proteins are involved in the assembly of active SCF complexes. 487

488 In this study, we integrated multiple levels of evidence to better describe the functional 489 constraints of individual ASK members. Orthology comparisons revealed that $64 \%$ of intronless 490 ASK genes were under neutral changes indicative of non-functionalization (Table 1). Both 491 developmental and population expression comparisons suggested that most intronless ASK 492 members have a very low expression level (Figure 2). In addition, sequence polymorphism 493 analysis showed a significant enrichment of intronless ASK members in the groups whose 494 mutations were under neutral changes (Figure 3C, clades I and II). Collectively, these data 495 suggest that most, if not all, retroposed ASK members are under low functional constraints.

496 This is indeed not surprising, since retroposed genes have a much high rate of 497 pseudogenization, as suggested in the duplication studies of the human genome (Torrents et al. 498 2003). Consistently, our previous evolutionary studies of the F-box gene superfamily also 499 discovered a significant enrichment of intronless genes in the pseudogene group (Hua et al. 500 2011). It will be of interest to explore the role of retroposition on the expansion of intronless $F$ 501 box genes.

502

503 The large expression variance of ASK1 may indicate diverse functions of SCF complexes 504 Expression comparisons revealed a greater than 430 RPKM ( 10 fold) variance in ASK1 505 transcript levels among individual $A$. thaliana accessions (Figure 2). Sequence analyses further 506 suggested the presence of a putative transcriptional repressor that might contribute to such 507 large variations in expression. Upstream sequences with more variable sites may prevent the 508 binding of this transcriptional repressor, thus increasing expression. The finding that more 509 polymorphic upstream sequences result in higher expression may support this model (Figure 4).

510 Such large variation in ASK1 expression among natural variants suggests that ASK1 is either 511 very effective in promoting the assembly of SCF complexes or is involved in an as-yet-unknown 512 mechanism to regulate the polymorphic functions of SCF complexes. It will be noteworthy to 513 further investigate the proteomic variance of SCF-mediated protein ubiquitylation in the future. 514

\section{Conclusions}


516 In this study, our improved phylogenetic analysis resolved the inconsistency between the

517 phylogeny of ASK genes and the single origin of retroposed ASK members (Kong et al. 2007).

518 Through evolutionary selection analysis and sequence polymorphism comparison, we

519 discovered both adaptive and degenerative evolutionary processes in the ASK family. Yeast

520 two-hybrid quantitative interaction assay and expression analysis across different accessions

521 further indicated that recent neutral changes in the ASK2 coding sequence likely weakened its

522 interactions with F-box proteins and that highly polymorphic upstream regions of $A S K 1$ may

523 contribute to adaptive roles of SCF complexes in Arabidopsis, respectively.

524

\section{Supplemental Information}

526 Table S1. OrthoMCL groups of Skp1 proteins in A. thaliana, A. lyrata, and A. halleri

527 Table S2. Gene identifications of 15 known F-box proteins selected for testing the differential

528 interactions with ASK1 and ASK2

529 Table S3. Raw data for $\beta$-galactosidase activity assay

530 File S1. Amino acid sequences of the Skp1 proteins annotated in A. thaliana, A. lyrata, and $A$.

531 halleri

532 Figure S1. An improved amino acid sequence alignment of 47 Arabidopsis Skp1 protein

533 sequences

534 Figure S2. A GeneWise reannotation uncovers a frame-shift mutation in ASK6.

535 Figure S3. A GeneWise reannotation uncovers a frame-shift mutation in AlySkp02.

536 Figure S4. A GeneWise reannotation uncovers a frame-shift mutation in AhaSkp11. 


\section{Figure Legends}

538 Figure 1. A short evolutionary history of the Arabidopsis Skp1 genes within the Arabidopsis

539 genus. A) Phylogenetic relationships of the ASK members. Intronic genes are highlighted in

540 red. Scale bar, average substitutions per site. B) An improved phylogenetic analysis reveals

541 one single origin of retroposed Skp1 genes in the Arabidopsis genus. a-e, 5 ancient retroposed

542 loci produced by the transcripts of the highly expressed ancestor locus of ASK1. 1-11, 11

543 clades shaded with dark and gray color showing clear orthologous relationships among the

544 three Arabidopsis species. The tree was generated by a maximum likelihood method.

545 Statistical significance equal to or greater than $50 \%$ of 1,000 bootstrap re-samplings is indicated

546 in each node. S: segmental duplication; T: tandem duplication; R: retroposed duplication. The

547 ID number from the original annotation is indicated if a Skp1 gene is duplicated through tandem

548 duplications or described in File S1. Intronic Skp1 genes and scale bar are described as in A.

549 Species abbreviations: A. halleri (Aha), A. lyrata (Aly), and A. thaliana (Ath). C) Birth-and-death

550 history of Skp1 genes in the Arabidopsis genus. + and - indicate gain and loss of Skp1

551 members, respectively. Species abbreviations are as in B.

552

553 Figure 2. Expression variation of $A S K$ genes. A) Variation of absolute expression throughout a

554 developmental time course. B) A heatmap analysis of mean expression variation of $A S K$ genes

555 across 8 tissues/organs. C) Expression variation of ASK genes in leaves of 144 accessions.

556 The width of each boxplot is proportional to the number of accessions having a non-zero

557 expression data of the corresponding ASK gene. D) Expression comparison of ASK1 and ASK2

558 in Col-0, Ler-0, and Ws-0.

559

560 Figure 3. Sequence polymorphic comparison among different $A S K$ genes. A) Frequency

561 distribution of rare ASK alleles with non-synonymous and synonymous mutations. B) Variation

562 of sequence polymorphisms in three regions of an ASK gene. UP: 500 bp upstream of start

563 codon, CDS: coding sequence, DN: 500 bp downstream of the stop codon. C) A heatmap

564 representation of Tajima's $D$ values demonstrating the differential evolutionary constraints of

565 polymorphic mutations among ASK genes. Asterisks indicate a significantly low Tajima's D

566 value that deviates from neutral mutations $(p<0.05)$.

567

568 Figure 4. Contribution of sequence polymorphism to gene expression. A) Latitudinal variation

569 of $A S K 1$ expression. Asterisks indicate that the mean expression of the indicated group is

570 significantly lower than that of the group within latitudes $45-50^{\circ} \mathrm{N}$ (Wilcoxon rank-sum test, $p<$ 
571 0.01). B) A window-slide polymorphic comparison of a $2.5 \mathrm{~kb}$ region upstream of the

572 transcriptional start site of $A S K 1$ among populations from 3 latitudinal regions as in $\mathrm{A}$. C) $\mathrm{A}$

573 window-slide polymorphic comparison of a group of test populations from 3 latitudinal regions,

574 performed as in B. D) A window-slide polymorphic comparison of three groups of populations

575 with low, medium, and high expression levels of ASK1 that was performed as in B.

576

577 Figure 5. Quantitative interaction assay of ASK1 and ASK2 with 15 selected known F-box

578 proteins. The accession IDs of F-box proteins are listed in Table S2. A) Growth of yeast cells

579 expressing each indicated pair of bait and prey proteins on SD-Leu-Trp-Ade-His+X- $\alpha$-gal

580 medium. B) Control growth of the corresponding yeast cells in A on SD-Leu-Trp medium.

581 Yeast cells were grown on media at $30^{\circ} \mathrm{C}$ for an indicated time period and their growth was

582 subsequently recorded by scanning the plates with a Canon 9000F Mark II scanner. B)

583 Quantification of pairwise ASK1 and ASK2 interactions with $15 \mathrm{~F}$-box proteins as shown in A.

584 The interaction strength of each pair of bait and prey proteins shown in $A$ was normalized by the

585 control growth strength of the corresponding yeast cells in B. C) $\beta$-galactosidase activity assay.

586 The mated yeast cells expressing the indicated pair of bait and prey proteins were grown on

587 SD-Leu-Trp medium and used for the assay. The $\beta$-galactosidase activities shown are mean

588 values \pm SD measured from two independent assays.

589

590

591

592 
593

594

595

596

597

598

599

600

601

602

603

604

605

606

607

608

609

610

611

612

613

614

615

616

617

618

619

620

621

622

623

624

625

626

\section{References}

Altschul SF, Gish W, Miller W, Myers EW, and Lipman DJ. 1990. Basic local alignment search tool. J Mol Biol 215:403-410. 10.1016/S0022-2836(05)80360-2

Birney E, Clamp M, and Durbin R. 2004. GeneWise and Genomewise. Genome Res 14:988995. 10.1101/gr.1865504

Cao J, Schneeberger K, Ossowski S, Gunther T, Bender S, Fitz J, Koenig D, Lanz C, Stegle O, Lippert C, Wang X, Ott F, Muller J, Alonso-Blanco C, Borgwardt K, Schmid KJ, and Weigel D. 2011. Whole-genome sequencing of multiple Arabidopsis thaliana populations. Nat Genet 43:956-963. 10.1038/ng.911

Capella-Gutierrez S, Silla-Martinez JM, and Gabaldon T. 2009. trimAl: a tool for automated alignment trimming in large-scale phylogenetic analyses. Bioinformatics 25:1972-1973. 10.1093/bioinformatics/btp348

Chen K, Durand D, and Farach-Colton M. 2000. NOTUNG: a program for dating gene duplications and optimizing gene family trees. J Comput Biol 7:429-447. $10.1089 / 106652700750050871$

Consortium G. 2016. 1,135 genomes reveal the global pattern of polymorphism in Arabidopsis thaliana. Cell 166:481-491. 10.1016/j.cell.2016.05.063

Edgar RC. 2004. MUSCLE: multiple sequence alignment with high accuracy and high throughput. Nucleic Acids Res 32:1792-1797. 10.1093/nar/gkh340

Farras R, Ferrando A, Jasik J, Kleinow T, Okresz L, Tiburcio A, Salchert K, del Pozo C, Schell $J$, and Koncz C. 2001. SKP1-SnRK protein kinase interactions mediate proteasomal binding of a plant SCF ubiquitin ligase. EMBO $\mathrm{J}$ 20:2742-2756. 10.1093/emboj/20.11.2742

Finley D, Ulrich HD, Sommer T, and Kaiser P. 2012. The ubiquitin-proteasome system of Saccharomyces cerevisiae. Genetics 192:319-360. 10.1534/genetics.112.140467

Gagne JM, Downes BP, Shiu SH, Durski AM, and Vierstra RD. 2002. The F-box subunit of the SCF E3 complex is encoded by a diverse superfamily of genes in Arabidopsis. Proc Natl Acad Sci USA 99:11519-11524. 10.1073/pnas.162339999

Gan X, Stegle O, Behr J, Steffen JG, Drewe P, Hildebrand KL, Lyngsoe R, Schultheiss SJ, Osborne EJ, Sreedharan VT, Kahles A, Bohnert R, Jean G, Derwent P, Kersey P, Belfield EJ, Harberd NP, Kemen E, Toomajian C, Kover PX, Clark RM, Ratsch G, and Mott R. 2011. Multiple reference genomes and transcriptomes for Arabidopsis thaliana. Nature 477:419-423. 10.1038/nature10414 
627 Hu TT, Pattyn P, Bakker EG, Cao J, Cheng JF, Clark RM, Fahlgren N, Fawcett JA, Grimwood J, 628 Gundlach H, Haberer G, Hollister JD, Ossowski S, Ottilar RP, Salamov AA,

629

630

631

632

633

634

635

636

637

638

639

640

641

642

643

644

645

646

647

648

649

650

651

652

653

654

655

656

657

658

659 Schneeberger K, Spannagl M, Wang X, Yang L, Nasrallah ME, Bergelson J, Carrington JC, Gaut BS, Schmutz J, Mayer KF, Van de Peer Y, Grigoriev IV, Nordborg M, Weigel D, and Guo YL. 2011. The Arabidopsis lyrata genome sequence and the basis of rapid genome size change. Nat Genet 43:476-481. 10.1038/ng.807

Hua Z, Doroodian P, and Vu W. 2018. Contrasting duplication patterns reflect functional diversities of ubiquitin and ubiquitin-like protein modifiers in plants. Plant J 95:296-311. 10.1111/tpj. 13951

Hua Z, and Early MJ. 2018. Closing Target Trimming: a Perl package for discovering hidden superfamily loci in genomes. bioRxiv:490490. 10.1101/490490

Hua Z, and Kao TH. 2006. Identification and characterization of components of a putative petunia S-locus F-box-containing E3 ligase complex involved in S-RNase-based selfincompatibility. Plant Cell 18:2531-2553. 10.1105/tpc.106.041061

Hua Z, Pool JE, Schmitz RJ, Schultz MD, Shiu SH, Ecker JR, and Vierstra RD. 2013. Epigenomic programming contributes to the genomic drift evolution of the F-Box protein superfamily in Arabidopsis. Proc Natl Acad Sci USA 110:16927-16932.

\subsection{3/pnas.1316009110}

Hua Z, and Vierstra RD. 2011. The cullin-RING ubiquitin-protein ligases. Annu Rev Plant Biol 62:299-334. 10.1146/annurev-arplant-042809-112256

Hua Z, Zou C, Shiu SH, and Vierstra RD. 2011. Phylogenetic comparison of F-Box (FBX) gene superfamily within the plant kingdom reveals divergent evolutionary histories indicative of genomic drift. PLoS One 6:e16219. 10.1371/journal.pone.0016219

Hurles M. 2004. Gene duplication: the genomic trade in spare parts. PLoS Biol 2:E206. 10.1371/journal.pbio.0020206

Katoh K, Rozewicki J, and Yamada KD. 2017. MAFFT online service: multiple sequence alignment, interactive sequence choice and visualization. Brief Bioinform. 10.1093/bib/bbx108

Kim JD, Faulk C, and Kim J. 2007. Retroposition and evolution of the DNA-binding motifs of YY1, YY2 and REX1. Nucleic Acids Res 35:3442-3452. 10.1093/nar/gkm235

Koch MA, and Kiefer M. 2005. Genome evolution among cruciferous plants: a lecture from the comparison of the genetic maps of three diploid species - Capsella rubella, Arabidopsis lyrata subsp. petraea, and A. thaliana. Am J Bot 92:761-767. 10.3732/ajb.92.4.761 
660 Kong H, Landherr LL, Frohlich MW, Leebens-Mack J, Ma H, and dePamphilis CW. 2007.

661

662

663

664

665

666

667

668

669

670

671

672

673

674

675

676

677

678

679

680

681

682

683

684

685

686

687

688

689

690

691

Patterns of gene duplication in the plant SKP1 gene family in angiosperms: evidence for multiple mechanisms of rapid gene birth. Plant $J$ 50:873-885. 10.1111/j.1365313X.2007.03097.x

Kong $\mathrm{H}$, Leebens-Mack J, Ni W, dePamphilis CW, and Ma H. 2004. Highly heterogeneous rates of evolution in the SKP1 gene family in plants and animals: functional and evolutionary implications. Mol Biol Evol 21:117-128. 10.1093/molbev/msh001

Kuroda H, Yanagawa Y, Takahashi N, Horii Y, and Matsui M. 2012. A comprehensive analysis of interaction and localization of Arabidopsis SKP1-like (ASK) and F-box (FBX) proteins. PLoS One 7:e50009. 10.1371/journal.pone.0050009

Li L, Stoeckert CJ, Jr., and Roos DS. 2003. OrthoMCL: identification of ortholog groups for eukaryotic genomes. Genome Research 13:2178-2189. 10.1101/gr.1224503

Li Z, Defoort J, Tasdighian S, Maere S, Van de Peer Y, and De Smet R. 2016. Gene duplicability of core genes is highly consistent across all angiosperms. Plant Cell 28:326344. 10.1105/tpc. 15.00877

Long Q, Rabanal FA, Meng D, Huber CD, Farlow A, Platzer A, Zhang Q, Vilhjalmsson BJ, Korte A, Nizhynska V, Voronin V, Korte P, Sedman L, Mandakova T, Lysak MA, Seren U, Hellmann I, and Nordborg M. 2013. Massive genomic variation and strong selection in Arabidopsis thaliana lines from Sweden. Nat Genet 45:884-890. 10.1038/ng.2678

Miller JH. 1972. Experiments in Molecular Genetics. Cold Spring Harbor, NY: Cold Spring Harbor Laboratory Press.

Nei M, and Kumar S. 2000. Molecular evolution and phylogenetics. Oxford University Press, Oxford.

Nekrutenko A, Makova KD, and Li WH. 2002. The $K_{A} / K_{S}$ ratio test for assessing the proteincoding potential of genomic regions: an empirical and simulation study. Genome Res 12:198-202. 10.1101/gr.200901

Nozawa M, Kawahara Y, and Nei M. 2007. Genomic drift and copy number variation of sensory receptor genes in humans. Proc Natl Acad Sci USA 104:20421-20426. 10.1073/pnas.0709956104

Ohno S. 1970. Evolution by gene duplication. Springer-Verlag, New York.

Panchy N, Lehti-Shiu M, and Shiu SH. 2016. Evolution of gene duplication in plants. Plant Physiol 171:2294-2316. 10.1104/pp.16.00523 
692 Rawat V, Abdelsamad A, Pietzenuk B, Seymour DK, Koenig D, Weigel D, Pecinka A, and

693

694

695

696

697

698

699

700

701

702

703

704

705

706

707

708

709

710

711

712

713

714

715

716

717

718

719

720

721

722

723

724

725

Schneeberger K. 2015. Improving the annotation of Arabidopsis lyrata using RNA-Seq data. PLoS One 10:e0137391. 10.1371/journal.pone.0137391

Schmitz RJ, Schultz MD, Urich MA, Nery JR, Pelizzola M, Libiger O, Alix A, McCosh RB, Chen $\mathrm{H}$, Schork NJ, and Ecker JR. 2013. Patterns of population epigenomic diversity. Nature 495:193-198. 10.1038/nature11968

Shabek N, and Zheng N. 2014. Plant ubiquitin ligases as signaling hubs. Nat Struct Mol Biol 21:293-296. 10.1038/nsmb.2804

Stamatakis A. 2014. RAxML version 8: a tool for phylogenetic analysis and post-analysis of large phylogenies. Bioinformatics 30:1312-1313. 10.1093/bioinformatics/btu033

Tajima F. 1989. Statistical method for testing the neutral mutation hypothesis by DNA polymorphism. Genetics 123:585-595.

Taly JF, Magis C, Bussotti G, Chang JM, Di Tommaso P, Erb I, Espinosa-Carrasco J, Kemena C, and Notredame C. 2011. Using the T-Coffee package to build multiple sequence alignments of protein, RNA, DNA sequences and 3D structures. Nat Protoc 6:16691682. 10.1038/nprot.2011.393

Torrents D, Suyama M, Zdobnov E, and Bork P. 2003. A genome-wide survey of human pseudogenes. Genome Res 13:2559-2567. 10.1101/gr.1455503

Vierstra RD. 2009. The ubiquitin-26S proteasome system at the nexus of plant biology. Nat Rev Mol Cell Biol 10:385-397. 10.1038/nrm2688

Wang Z, Gerstein M, and Snyder M. 2009. RNA-Seq: a revolutionary tool for transcriptomics. Nat Rev Genet 10:57-63. 10.1038/nrg2484

Xu G, Ma H, Nei M, and Kong H. 2009. Evolution of F-box genes in plants: different modes of sequence divergence and their relationships with functional diversification. Proc Natl Acad Sci USA 106:835-840. 10.1073/pnas.0812043106

Yang M, Hu Y, Lodhi M, McCombie WR, and Ma H. 1999. The Arabidopsis SKP1-LIKE1 gene is essential for male meiosis and may control homologue separation. Proc Natl Acad Sci USA 96:11416-11421.

Yang X, Kalluri UC, Jawdy S, Gunter LE, Yin T, Tschaplinski TJ, Weston DJ, Ranjan P, and Tuskan GA. 2008. The F-box gene family is expanded in herbaceous annual plants relative to woody perennial plants. Plant Physiol 148:1189-1200. 10.1104/pp.108.121921

Yang Z. 2007. PAML 4: phylogenetic analysis by maximum likelihood. Mol Biol Evol 24:15861591. $10.1093 / \mathrm{molbev} / \mathrm{msm} 088$

Peer) reviewing PDF | (2019:02:34819:1:1:NEW 2 Mar 2019) 
726 Yau R, and Rape M. 2016. The increasing complexity of the ubiquitin code. Nat Cell Biol 727 18:579-586. 10.1038/ncb3358

728 Zhao D, Ni W, Feng B, Han T, Petrasek MG, and Ma H. 2003. Members of the Arabidopsis729 SKP1-like gene family exhibit a variety of expression patterns and may play diverse $730 \quad$ roles in Arabidopsis. Plant Physiol 133:203-217.

731 Zheng N, Schulman BA, Song L, Miller JJ, Jeffrey PD, Wang P, Chu C, Koepp DM, Elledge SJ, 732 Pagano M, Conaway RC, Conaway JW, Harper JW, and Pavletich NP. 2002. Structure

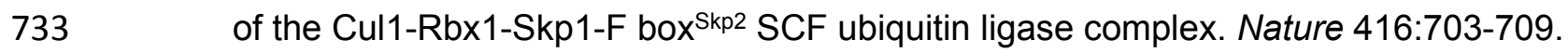

$734 \quad 10.1038 / 416703 a$

735 


\section{Table $\mathbf{1}$ (on next page)}

Maximum likelihood test of neutral evolution by comparing neutral evolutionary model (ML1: dN/dS =1) and non-neutral evolutionary model (ML2: free $\mathrm{dN} / \mathrm{dS}$ value) of ASK genes. 
1 Table 1. Maximum likelihood test of neutral evolution by comparing neutral evolutionary model

2 (ML1: dN/dS=1) and non-neutral evolutionary model (ML2: free dN/dS value) of $A S K$ genes

\begin{tabular}{lllllllll}
\hline Ath Skps & Aly Skps & $\mathrm{dS}$ & $\mathrm{dN}$ & $\mathrm{dN} / \mathrm{dS}$ & InML1 & InML2 & $2 \Delta I n \mathrm{ML}$ & Selection* \\
\hline ASK2 & AlySkp05 & 0.2 & 0.0 & 0.1 & -799.9 & -779.5 & 40.8 & Non-neutral \\
ASK4 & AlySkp04 & 0.3 & 0.0 & 0.1 & -810.3 & -792.4 & 35.8 & Non-neutral \\
ASK3 & AlySkp04 & 0.3 & 0.0 & 0.1 & -793.3 & -778.7 & 29.1 & Non-neutral \\
ASK1 & AlySkp01 & 0.2 & 0.0 & 0.1 & -759.6 & -745.3 & 28.6 & Non-neutral \\
ASK18 & AlySkp08 & 0.2 & 0.1 & 0.3 & -916.5 & -908.8 & 15.5 & Non-neutral \\
ASK6 & AlySkp02 & 0.3 & 0.1 & 0.3 & -463.8 & -460.0 & 7.5 & Non-neutral \\
ASK11 & AlySkp06 & 0.2 & 0.1 & 0.4 & -776.3 & -773.8 & 5.0 & Non-neutral \\
ASK12 & AlySkp06 & 0.2 & 0.1 & 0.5 & -779.2 & -777.2 & 4.0 & Non-Neutral \\
ASK13 & AlySkp13 & 0.2 & 0.1 & 0.6 & -831.1 & -829.8 & 2.6 & Neutral \\
ASK14 & AlySkp15 & 0.3 & 0.2 & 0.6 & -836.6 & -835.5 & 2.2 & Neutral \\
ASK8 & AlySkp09 & 0.2 & 0.3 & 1.6 & -909.0 & -908.0 & 2.0 & Neutral \\
ASK10 & AlySkp09 & 0.2 & 0.2 & 1.4 & -880.8 & -880.4 & 0.9 & Neutral \\
ASK19 & AlySkp03 & 0.2 & 0.2 & 0.8 & -1110.3 & -1109.9 & 0.8 & Neutral \\
ASK9 & AlySkp09 & 0.2 & 0.2 & 0.8 & -892.6 & -892.5 & 0.3 & Neutral \\
ASK16 & AlySkp12 & 0.1 & 0.1 & 0.9 & -885.8 & -885.8 & 0.1 & Neutral \\
ASK17 & AlySkp07 & 0.1 & 0.1 & 1.1 & -779.6 & -779.6 & 0.1 & Neutral \\
ASK5 & AlySkp10 & 0.1 & 0.1 & 0.9 & -804.5 & -804.4 & 0.1 & Neutral \\
ASK7 & AlySkp09 & 0.2 & 0.2 & 1.1 & -740.1 & -740.1 & 0.1 & Neutral \\
\hline
\end{tabular}

$3{ }^{*} x^{2}(p=0.05, d f=1)=2.71$ (Yang 2007). Abbreviations: A. thaliana (Ath) and A. lyrata (Aly). 


\section{Figure 1 (on next page)}

A short evolutionary history of the Arabidopsis Skpl genes within the Arabidopsis genus.

A) Phylogenetic relationships of the ASK members. Intronic genes are highlighted in red.

Scale bar, average substitutions per site. B) An improved phylogenetic analysis reveals one single origin of retroposed Skp1 genes in the Arabidopsis genus. a-e, 5 ancient retroposed loci produced by the transcripts of the highly expressed ancestor locus of ASK1. 1-11, 11 clades shaded with dark and gray color showing clear orthologous relationships among the three Arabidopsis species. The tree was generated by a maximum likelihood method. Statistical significance equal to or greater than $50 \%$ of 1,000 bootstrap re-samplings is indicated in each node. S: segmental duplication; T: tandem duplication; R: retroposed duplication. The ID number from the original annotation is indicated if a Skpl gene is duplicated through tandem duplications or described in File S1. Intronic Skp1 genes and scale bar are described as in A. Species abbreviations: $A$. halleri(Aha), $A$. lyrata $(A / y)$, and $A$. thaliana(Ath). C) Birth-and-death history of $S k p 1$ genes in the Arabidopsis genus. + and indicate gain and loss of Skp1 members, respectively. Species abbreviations are as in B. 
A



C

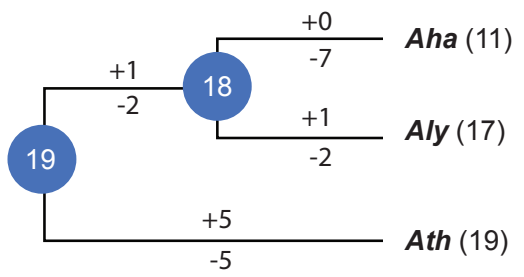

B




Figure 2 (on next page)

Expression variation of ASK genes.

A) Variation of absolute expression throughout a developmental time course. B) A heatmap analysis of mean expression variation of ASK genes across 8 tissues/organs. C) Expression variation of ASK genes in leaves of 144 accessions. The width of each boxplot is proportional to the number of accessions having a non-zero expression data of the corresponding ASK gene. D) Expression comparison of ASK1 and ASK2 in Col-0, Ler-0, and Ws-0. 
A

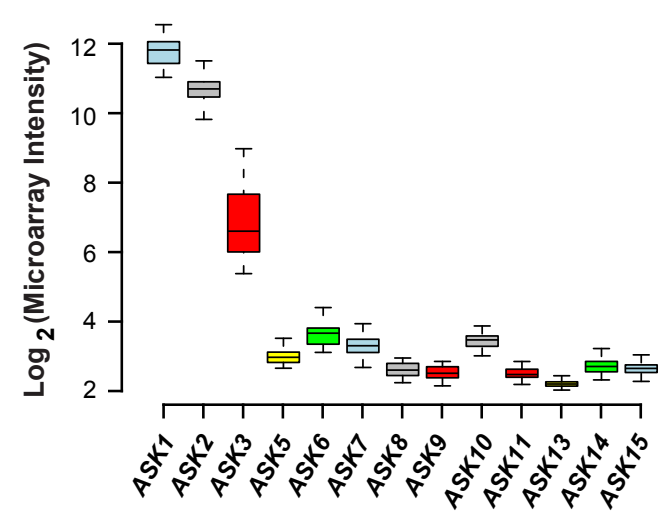

C

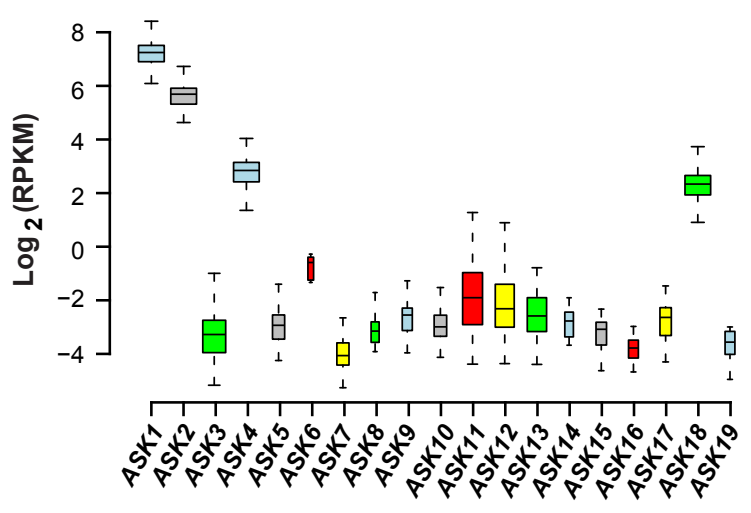

B

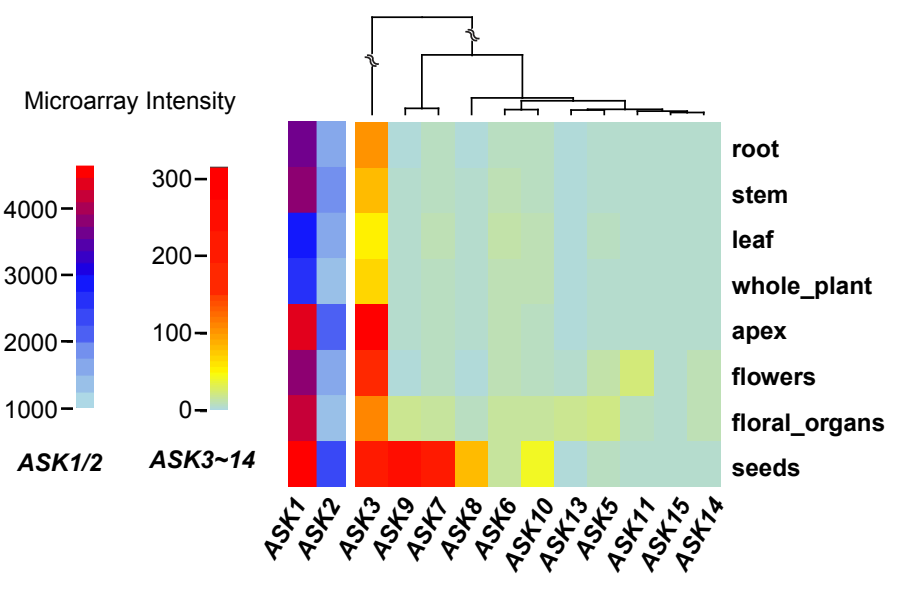

D

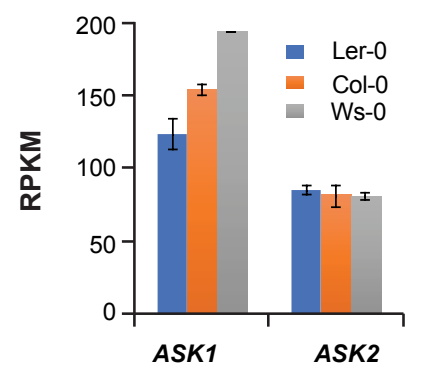


Figure 3 (on next page)

Sequence polymorphic comparison among different ASK genes.

A) Frequency distribution of rare ASK alleles with non-synonymous and synonymous

mutations. B) Variation of sequence polymorphisms in three regions of an ASK gene. UP: 500 bp upstream of start codon, CDS: coding sequence, DN: 500 bp downstream of the stop codon. C) A heatmap representation of Tajima's D values demonstrating the differential evolutionary constraints of polymorphic mutations among ASK genes. Asterisks indicate a significantly low Tajima's D value that deviates from neutral mutations $(p<0.05)$. 
A

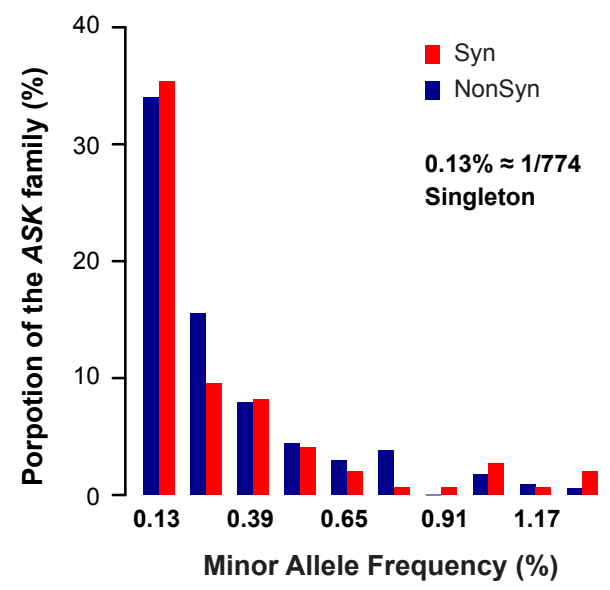

B



C

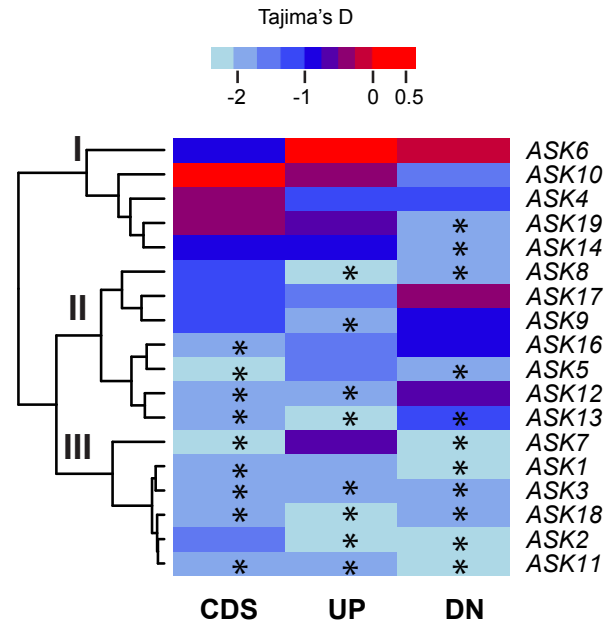


Figure 4 (on next page)

Contribution of sequence polymorphism to gene expression.

A) Latitudinal variation of $A S K 1$ expression. Asterisks indicate that the mean expression of the indicated group is significantly lower than that of the group within latitudes $45-50^{\circ} \mathrm{N}$ (Wilcoxon rank-sum test, $p<0.01$ ). B) A window-slide polymorphic comparison of a $2.5 \mathrm{~kb}$ region upstream of the transcriptional start site of ASK1 among populations from 3 latitudinal regions as in A. C) A window-slide polymorphic comparison of a group of test populations from 3 latitudinal regions, performed as in B. D) A window-slide polymorphic comparison of three groups of populations with low, medium, and high expression levels of ASK1 that was performed as in B. 
A

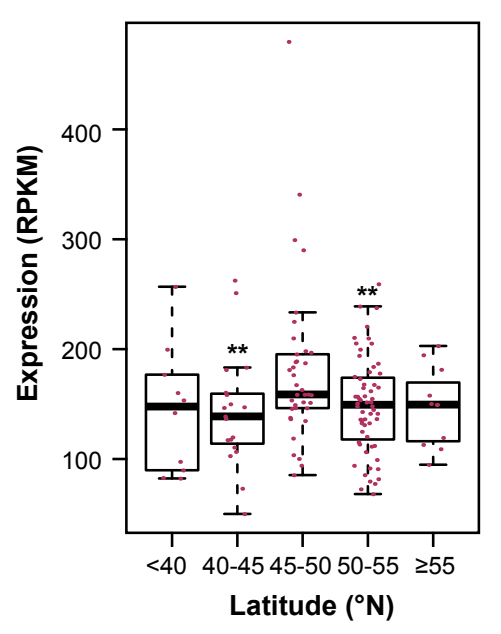

C

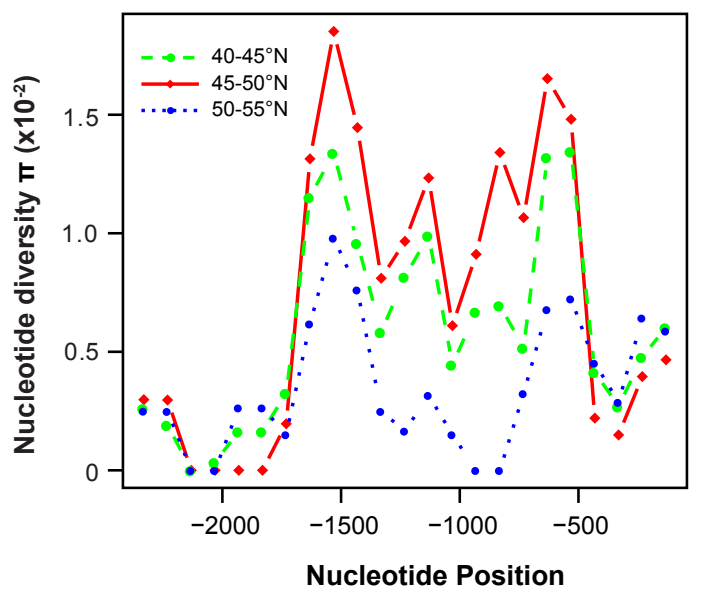

B

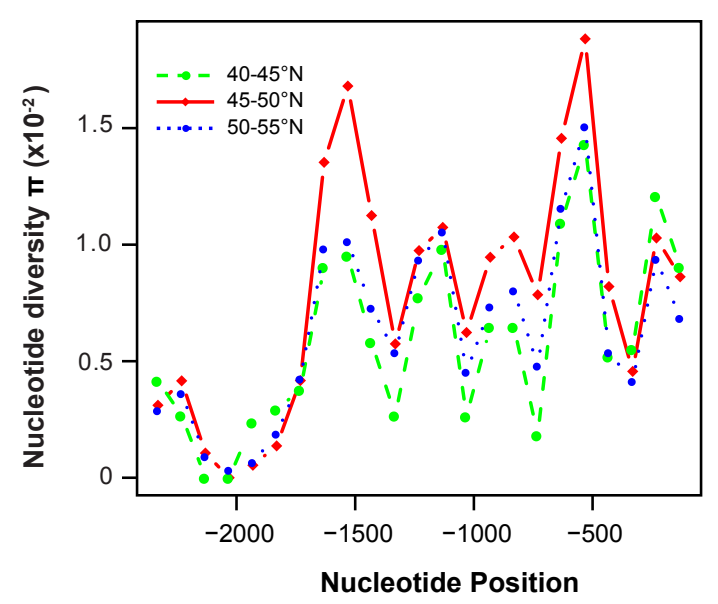

D






\section{Figure 5}

Quantitative interaction assay of ASK1 and ASK2 with 15 selected known F-box proteins.

The accession IDs of F-box proteins are listed in Table S2. A) Growth of yeast cells expressing each indicated pair of bait and prey proteins on SD-Leu-Trp-Ade-His+X-alpha-gal medium. B) Control growth of the corresponding yeast cells in A on SD-Leu-Trp medium. Yeast cells were grown on media at $30^{\circ} \mathrm{C}$ for an indicated time period and their growth was subsequently recorded by scanning the plates with a Canon 9000F Mark II scanner. B) Quantification of pairwise ASK1 and ASK2 interactions with $15 \mathrm{~F}$-box proteins as shown in A. The interaction strength of each pair of bait and prey proteins shown in A was normalized by the control growth strength of the corresponding yeast cells in B. C) Beta-galactosidase activity assay. The mated yeast cells expressing the indicated pair of bait and prey proteins were grown on SD-Leu-Trp medium and used for the assay. The beta-galactosidase activities shown are mean values \pm SD measured from two independent assays. 
A

B
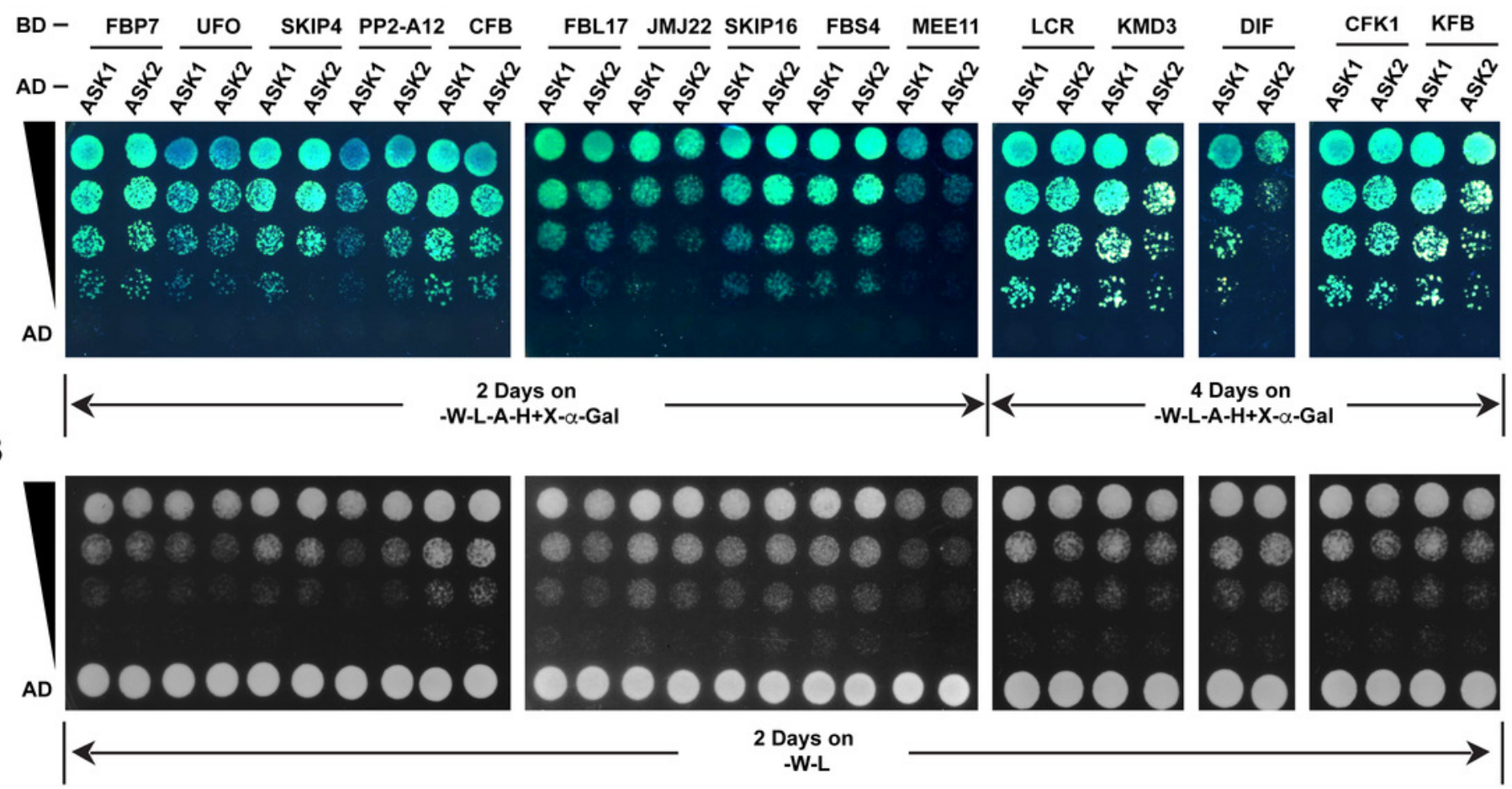

C



D

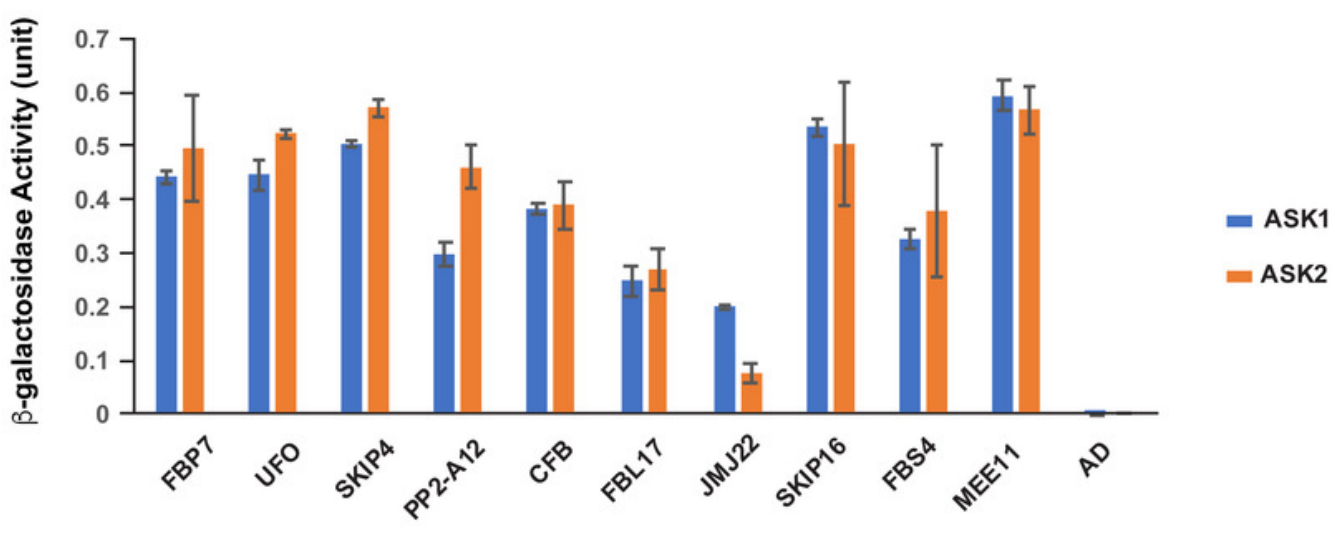

